\title{
A projective characterization of a class of abelian groups
}

\author{
Patrick W. KeEF
}

(Received August 26, 2013)

\begin{abstract}
This paper considers the class of abelian groups that are extensions of subgroups that are direct sums of cyclic groups by factor groups that are also of this form. This class is shown to be the projectives with respect to a natural collection of short exact sequences, and that the corresponding class of injectives consists of those groups whose first Ulm subgroup is pure-injective. This class of projectives is quite extensive, but satisfactory descriptions are given for the countable groups in the class that are either torsion-free, or else mixed groups of torsion-free rank one. Particular attention is paid to the behavior of the groups in these classes under localization at some prime.
\end{abstract}

Key words: purity, injectives, projectives, direct sums of cyclics, Ulm subgroups.

\section{Introduction}

By the term "group" we will mean an abelian group. Our terminology will, in the main, be standard, and will be consistent with that found in [2] or [3]. We will also employ a smattering of set theory for which we refer the reader to $[1]$.

If $G$ is a group, then the $\mathbb{Z}$-adic topology on $G$ uses $\{n G\}_{n \in \mathbb{N}}$ as a neighborhood base for 0 . The closure of $\{0\}$ in this topology, $\overline{\{0\}}=\cap_{n \in \mathbb{N}} n G$, is referred to as the (first) Ulm subgroup of $G$ and is written $G^{1}$.

If $B$ is a subgroup of $G$ and $A=G / B$, then $B$ is pure in $G$ iff for all $n \in \mathbb{N}$ we have $n B=B \cap n G$. The corresponding collection of pure short exact sequences agrees with the $\operatorname{Ulm}$ subgroup $\operatorname{Ext}(A, B)^{1}$. A group is pureinjective iff it is the direct sum of a divisible group and a (reduced) group that is complete in the $\mathbb{Z}$-adic topology (i.e., it is algebraically compact). A group is pure-projective iff it is $\Sigma$-cyclic (i.e., the direct sum of a collection of cyclic groups - we will also use $\Sigma_{f}$-cyclic to indicate a group that is isomorphic to the direct sum of a collection of finite cyclic groups).

In this paper we will investigate a parallel collection of ideas. We define the $\mathbb{Z}^{2}$-adic topology on the group $G$ by using $\left\{n G^{1}=n\left(G^{1}\right): n \in \mathbb{N}\right\}$ as 
a neighborhood base of 0 . In this topology the smallest closed subgroup of $G$ is $\overline{\{0\}}=\cap_{n \in \mathbb{N}} n G^{1}$, which is referred to as the second Ulm subgroup of $G$ and is written $G^{2}$.

We will define a collection of short exact sequences, which we refer to as $L^{2}$-pure-exact, whose properties with respect to the $\mathbb{Z}^{2}$-adic topology parallel the properties of the pure-exact sequences with respect to the $\mathbb{Z}$-adic topology. Specifically, we will show:

(a) A group is $L^{2}$-pure-injective iff it is the direct sum of a divisible group and a group that is complete in the $\mathbb{Z}^{2}$-adic topology. There are enough $L^{2}$-pure-injectives (Proposition 2.8 and Corollary 2.9).

(b) A group is $L^{2}$-pure-projective iff it has a subgroup that is $\Sigma$-cyclic such that the corresponding factor group is also $\Sigma$-cyclic. There are enough $L^{2}$-pure-projectives (Theorem 2.10).

Summarizing the contents of the paper, in Section 2 we construct the class of $L^{2}$-pure-exact sequences and verify properties (a) and (b). This section could be shortened somewhat using ideas from [4]; however, the present treatment has several advantages: It is nearly self-contained, using nothing more than basic ideas from the theory of pure-exact sequences; and in addition, in [4] the emphasis was on groups localized at a single prime, whereas in this work the emphasis is on the collection of all primes.

Condition (a) actually gives a reasonably complete picture of the collection of $L^{2}$-pure-injectives. The remainder of this paper is a discussion of the class of $L^{2}$-pure-projectives mentioned in (b), i.e., the groups that are extensions of $\Sigma$-cyclic subgroups by $\Sigma$-cyclic factors.

Clearly, a complete classification of these groups is not possible. For example, even in the case of $p$-groups, in ([5, Proposition 3.1(b)]) it was noted that for any separable $p$-group $S$ whatsoever, there is a $L^{2}$-pureprojective $p$-group $A$ such that $A / p^{\omega} A \cong S \oplus C$, where $C$ is $\Sigma_{f}$-cyclic (in the terminology of that paper, $A$ is a weak $p^{\omega 2}$-projective, or a $p_{*}^{\omega 2}$-projective). This means that the collection of $p$-torsion $L^{2}$-pure-projectives is at least as complicated as the class of all separable $p$-groups. In particular, there will almost certainly never be a satisfactory description of even the class of torsion $L^{2}$-pure-projectives.

However, there are things we can say. For example, in Section 3 we concentrate on torsion-free $L^{2}$-pure-projectives. We show that if $A$ is a torsion-free $L^{2}$-pure-projective group, then it is locally free (i.e., for every prime $p$, the localization $A_{p} \stackrel{\text { def }}{=} A \otimes \mathbb{Z}_{p}$ is a free $\mathbb{Z}_{p}$-module - Theorem 3.3). In 
addition, we show that the converse also holds if $A$ has countable torsion-free rank. Interestingly, using some basic set theory (i.e., the diamond principle, which holds in the constructible universe, $\mathrm{V}=\mathrm{L}$ ), we provide an example to show that this converse does not hold when $A$ has uncountable torsion-free rank (Theorem 3.6).

In Section 4 we give a complete description of the countable groups of torsion-free rank one that are $L^{2}$-pure-projective (Theorem 4.1 ). It is perhaps worth noting that this result could easily be extended to any group of torsion-free rank one whose torsion subgroup is totally projective. Finally, several examples are constructed. For example, it is shown that there is a countable mixed group $A$ of torsion-free rank one that is not $L^{2}$-pureprojective, but for which each localization $A_{p}$ is $L^{2}$-pure-projective in the class of local $\mathbb{Z}_{p}$-modules (Example 4.3 ).

\section{2. $L^{2}$-Purity}

Recall that the completion of a group $G$ in the $\mathbb{Z}$-adic topology is the inverse $\operatorname{limit} \lim G / n G$. In [2] this limit is denoted by $\hat{G}$, but we will prefer the notation $\overleftarrow{L^{1}} G$. So there is a natural homomorphism $G \rightarrow L^{1} G$ whose kernel is $G^{1}$ and whose image is a pure subgroup of $L^{1} G$ that is dense in the $\mathbb{Z}$-adic topology on $L^{1} G$. Denote the cokernel of this map by $Q^{1} G$; so $Q^{1} G$ is divisible.

Similarly, we let $L^{2} G=\longleftarrow \lim G / n G^{1}$ be the completion of $G$ in the $\mathbb{Z}^{2}$ adic topology. So there is a homomorphism $\phi_{G}: G \rightarrow L^{2} G$ whose kernel is $G^{2}$. We will usually identify $G / G^{2}$ with its image in $L^{2} G$; let $Q^{2} G$ be the cokernel of this embedding. We can also identify the $\mathbb{Z}$-adic completion of $G^{1}$, i.e., $L^{1} G^{1}=\lim G^{1} / n G^{1}$, with a subgroup of $L^{2} G$. Therefore, there is a commutative diagram

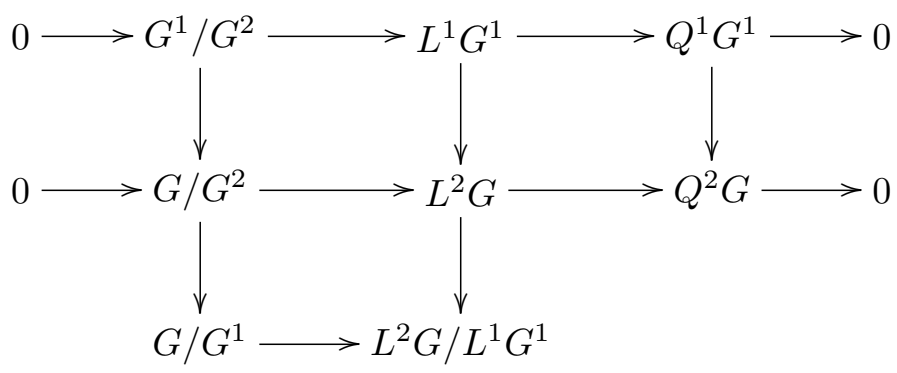


In this construction, it is readily verified that $G^{1} / G^{2}=\left(L^{1} G^{1}\right) \cap\left(G / G^{2}\right)$ and that $L^{2} G=\left(L^{1} G^{1}\right)+\left(G / G^{2}\right)$. This gives the following:

Lemma 2.1 If $G$ is a group, then there are natural isomorphisms

$$
\begin{gathered}
L^{2} G / L^{1} G^{1} \cong G / G^{1} \\
Q^{2} G=L^{2} G /\left(G / G^{2}\right) \cong L^{1} G^{1} /\left(G^{1} / G^{2}\right)=Q^{1} G^{1}
\end{gathered}
$$

In particular, the cokernel $Q^{2} G$ is divisible.

We now observe some additional consequences of the above relations. Since

(1) $G^{1} / G^{2}=\left(G / G^{2}\right)^{1} \subseteq\left(L^{2} G\right)^{1}$;

(2) $\left(L^{1} G^{1}\right) /\left(G^{1} / G^{2}\right)=Q^{1} G^{1}$ is divisible; and

(3) $\left(L^{2} G\right) /\left(L^{1} G^{1}\right) \cong G / G^{1}$,

it quickly follows that $L^{1} G^{1}=\left(L^{2} G\right)^{1}$. We expand these notions slightly as follows:

Proposition 2.2 If $G$ is a group and $n \in \mathbb{Z}$, then

(a) $\left(G / G^{2}\right) \cap n\left(L^{2} G\right)^{1}=n G^{1} / G^{2}$;

(b) $\left(G / G^{2}\right)+n\left(L^{2} G\right)^{1}=L^{2} G$;

(c) $L^{2} G / n\left(L^{2} G\right)^{1} \cong G / n G^{1}$.

Proof. Regarding (a), using the purity of $G^{1} / G^{2}$ in $L^{1} G^{1}$ we have

$$
\begin{aligned}
\left(G / G^{2}\right) \cap n\left(L^{2} G\right)^{1} & =\left(G / G^{2}\right) \cap n\left(L^{1} G^{1}\right) \\
& =\left(G / G^{2}\right) \cap\left(L^{1} G^{1}\right) \cap n\left(L^{1} G^{1}\right) \\
& =\left(G^{1} / G^{2}\right) \cap n\left(L^{1} G^{1}\right) \\
& =n G^{1} / G^{2} .
\end{aligned}
$$

As to (b), using the $\mathbb{Z}$-adic density of $G^{1} / G^{2}$ in $L^{1} G^{1}$ we have

$$
\begin{aligned}
\left(G / G^{2}\right)+n\left(L^{2} G\right)^{1} & =\left(G / G^{2}\right)+\left(G^{1} / G^{2}\right)+n\left(L^{1} G^{1}\right) \\
& =\left(G / G^{2}\right)+\left(L^{1} G^{1}\right)=L^{2} G .
\end{aligned}
$$

For (c), using (a) and (b) we have 


$$
\begin{aligned}
L^{2} G / n\left(L^{2} G\right)^{1} & =\left\{\left(G / G^{2}\right)+n\left(L^{2} G\right)^{1}\right\} / n\left(L^{2} G\right)^{1} \\
& \cong\left(G / G^{2}\right) /\left\{\left(G / G^{2}\right) \cap n\left(L^{2} G\right)^{1}\right\} \\
& =\left(G / G^{2}\right) /\left\{n G^{1} / G^{2}\right\} \\
& \cong G / n G^{1}
\end{aligned}
$$

The following important observation immediately follows from Proposition $2.2(\mathrm{c})$ :

Corollary 2.3 For any group $G$, there is a natural isomorphism $L^{2}\left(L^{2} G\right)$ $\cong L^{2} G$. In particular, $L^{2} G$ will always be complete in the $\mathbb{Z}^{2}$-adic topology.

We now use this functor to define a corresponding collection of short exact sequences.

Proposition 2.4 Suppose $E: 0 \rightarrow G \stackrel{\nu}{\rightarrow} X \rightarrow A \rightarrow 0$ is a short exact sequence. Then there is a homomorphism $f: X \rightarrow L^{2} G$ such that $\phi_{G}=f \circ \nu$ iff the induced sequence

$$
L^{2} E: 0 \rightarrow L^{2} G \stackrel{L^{2} \nu}{\longrightarrow} L^{2} X \longrightarrow L^{2} A \rightarrow 0
$$

is splitting exact.

Proof. Suppose first that $L^{2} E$ is splitting and let $\sigma: L^{2} X \rightarrow L^{2} G$ be such that $\sigma \circ\left(L^{2} \nu\right)$ is the identity on $L^{2} G$. If we let $f=\sigma \circ \phi_{X}: X \rightarrow L^{2} G$, then

$$
f \circ \nu=\sigma \circ \phi_{X} \circ \nu=\sigma \circ\left(L^{2} \nu\right) \circ \phi_{G}=\phi_{G},
$$

as required.

Suppose next that $f$ exists. It follows that there is a commutative diagram

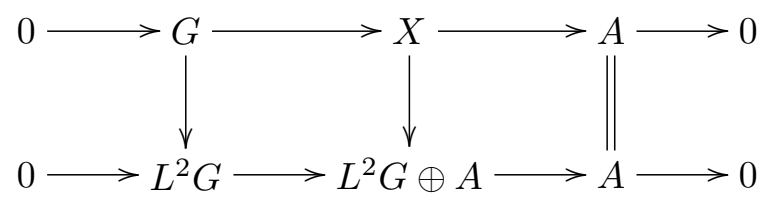

with splitting exact second row. For any $n \in \mathbb{N}$, this determines another commutative diagram 


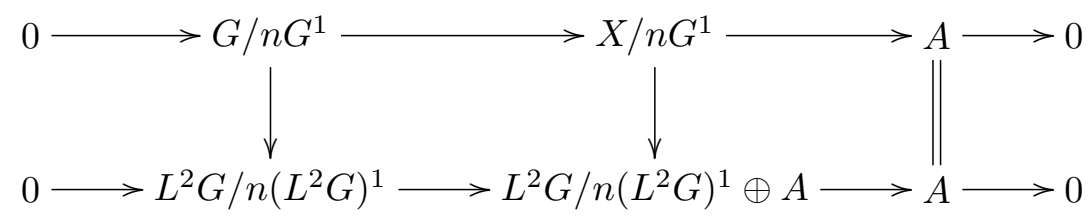

As the outer vertical homomorphisms are isomorphisms, so is the middle one. Since

$$
n X^{1} / n G^{1}=n\left(X / n G^{1}\right)^{1} \cong n\left(L^{2} G / n\left(L^{2} G\right)^{1} \oplus A\right)^{1},
$$

it follows that the middle vertical map determines an isomorphism

$$
\begin{aligned}
X / n X^{1} & \cong\left(X / n G^{1}\right) /\left(n X^{1} / n G^{1}\right) \\
& \cong\left(L^{2} G / n\left(L^{2} G\right)^{1} \oplus A\right) / n\left(L^{2} G / n\left(L^{2} G\right)^{1} \oplus A\right)^{1} \\
& \cong L^{2} G / n\left(L^{2} G\right)^{1} \oplus A / n A^{1} \cong G / n G^{1} \oplus A / n A^{1}
\end{aligned}
$$

Taking the inverse limit gives $L^{2} X \cong L^{2} G \oplus L^{2} A$, as stated.

As in Proposition 2.4, we say a short exact sequence $0 \rightarrow G \rightarrow X \rightarrow$ $A \rightarrow 0$ is $L^{2}$-pure iff the homomorphism $f: X \rightarrow L^{2} G$ exists iff the sequence represents an element of the kernel of the homomorphism

$$
\operatorname{Ext}(A, G) \rightarrow \operatorname{Ext}\left(A, L^{2} G\right)
$$

We will denote the collection of $L^{2}$-pure sequences by $L^{2} \operatorname{Ext}(A, G)$.

Using the functorial nature of the completion functor $L^{2}$, it is elementary to check that push-outs and pull-backs of $L^{2}$-pure sequences are themselves $L^{2}$-pure; so $L^{2} \operatorname{Ext}(A, G)$ is a bifunctor in $A$ and $G$. The next statement is an immediate consequence of this.

Proposition 2.5 Suppose $A$ is a subgroup of $B$ and $B$ is a subgroup of C.

(a) If $A$ is $L^{2}$-pure in $C$, then $A$ is $L^{2}$-pure in $B$.

(b) If $B$ is $L^{2}$-pure in $C$, then $B / A$ is $L^{2}$-pure in $C / A$.

We now observe a property of $L^{2}$-purity that is analogous to a similar property for ordinary purity. 
Corollary 2.6 If $0 \rightarrow G \rightarrow X \rightarrow A \rightarrow 0$ is $L^{2}$-pure, then for every $n \in \mathbb{N}$, the induced sequence

$$
0 \rightarrow G / n G^{1} \rightarrow X / n X^{1} \rightarrow A / n A^{1} \rightarrow 0
$$

is splitting exact.

Proof. $\quad$ By Proposition 2.2(c), this sequence is equivalent to

$$
0 \rightarrow\left(L^{2} G\right) / n\left(L^{2} G\right)^{1} \rightarrow\left(L^{2} X\right) / n\left(L^{2} X\right)^{1} \rightarrow\left(L^{2} A\right) / n\left(L^{2} A\right)^{1} \rightarrow 0
$$

which splits by Proposition 2.4.

Corollary 2.7 If $E$ is $L^{2}$-pure, then it is pure in the usual sense, and $G^{1}$ is pure in $X^{1}$.

With a wee bit more effort, using an analogue of ([2, Proposition 56.1]) and Corollary 2.6, we could verify that $L^{2} \operatorname{Ext}(A, G) \subseteq \operatorname{Ext}(A, G)^{2}$ for all groups $A$ and $G$. Unlike the case of ordinary purity, however, this containment can be proper.

We prove the next two statements simultaneously.

Proposition 2.8 The group $G$ is $L^{2}$-pure-injective iff it splits as $G \cong$ $C \oplus D$, where $D$ is divisible and $C$ is complete in the $\mathbb{Z}^{2}$-adic topology, iff $G^{1}$ is a pure-injective group.

Corollary 2.9 If $G$ is any group and $D$ is a divisible subgroup containing $G^{2}$, then there is a $L^{2}$-pure-injective resolution

$$
0 \rightarrow G \rightarrow L^{2} G \oplus D \rightarrow Q^{2} G \oplus E \rightarrow 0
$$

where $E$ is also divisible.

Proof. Suppose first that, as in Proposition 2.8, $G$ splits as $G \cong C \oplus$ $D$, where $D$ is divisible, and $C$ is complete in the $\mathbb{Z}^{2}$-adic topology; we intend to show that $G$ is $L^{2}$-pure-injective. Since divisible groups are just plain injective, there is no loss of generality in assuming that $D=\{0\}$ and $G=C$ is reduced and complete in the $\mathbb{Z}^{2}$-adic topology. In this case $\phi_{G}$ is an isomorphism, so for any $\operatorname{group} A, \operatorname{Ext}(A, G) \rightarrow \operatorname{Ext}\left(A, L^{2} G\right)$ is an isomorphism. And this implies that $L^{2} \operatorname{Ext}(A, G)=\{0\}$, i.e., $G$ is $L^{2}$-pureinjective. 
Next, note that if $G$ is any group, then the sequence in Corollary 2.9 clearly satisfies Proposition 2.4, so that it is $L^{2}$-pure. Since we have just shown that a group of the form $L^{2} G \oplus D$ must be $L^{2}$-pure-injective, we have verified Corollary 2.9.

Finally, if $G$ is an arbitrary $L^{2}$-pure-injective, then the sequence in Corollary 2.9 must split. But it is easy to see that if $H$ is a group (such as $\left.L^{2} G \oplus D\right)$ for which $H^{1}$ is pure-injective, then any summand of $H$ also has that property. Therefore, $G^{1}$ must be a pure-injective, and so $G$ is $L^{2}$-pure-injective.

Note that there is a push-out diagram

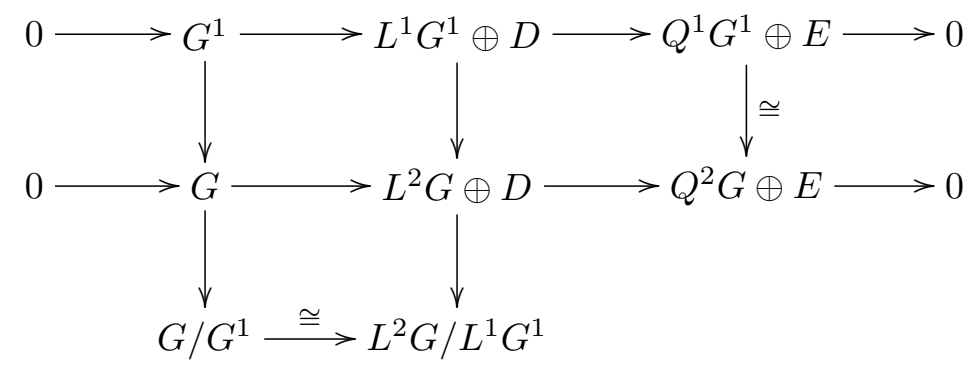

In other words, an $L^{2}$-pure-injective resolution of $G$ can be thought of as just a push-out of a pure-injective resolution of $G^{1}$.

The pure-injective (i.e., algebraically compact) groups can be completely described using cardinal invariants. Therefore, in some sense, the preceding discussion gives a pretty adequate description of the $L^{2}$-pure-injective groups. Of course, the Ulm factor $G / G^{1}$ can be nearly anything, and we do not have any control over the way the first Ulm subgroup and Ulm factor fit together to form the overall group; but at least it is clear what we can know and what we cannot.

We now turn to the main focus of our investigations, the corresponding collection of $L^{2}$-pure-projectives. We will use at several points the wellknown (and easily checked) fact that if $X$ is a subgroup of $Y$ such that $Y / X$ is $\Sigma$-cyclic, and $Z$ is any group, then any homomorphism $f: X \rightarrow Z^{1}$ extends to a homomorphism $Y \rightarrow Z$.

Theorem 2.10 For a group A, the following are equivalent:

(a) A is a $L^{2}$-pure-projective; 
(b) $A$ is an extension of a $\Sigma$-cyclic subgroup by a $\Sigma$-cyclic factor. In other words, $A$ has a $\Sigma$-cyclic subgroup $Z$ such that $A / Z$ is $\Sigma$-cyclic;

$\left(\mathrm{b}_{f}\right) A$ is an extension of a $\Sigma$-cyclic subgroup by a $\Sigma_{f}$-cyclic factor. In other words, $A$ has a $\Sigma$-cyclic subgroup $Z$ such that $A / Z$ is $\Sigma_{f}$-cyclic;

(c) $A$ can be embedded in a group of the form $Y=\bigoplus_{i \in I} B_{i}$, where for each $i \in I, B_{i}^{1}$ is cyclic and $B_{i} / B_{i}^{1}$ is $\Sigma_{f}$-cyclic.

There are enough $L^{2}$-pure-projectives.

Proof. We will begin by showing that $(\mathrm{b}),\left(\mathrm{b}_{f}\right)$ and $(\mathrm{c})$ are equivalent. Suppose first that (c) holds, and that $A \subseteq Y$ is as stated; we will then prove $\left(\mathrm{b}_{f}\right)$. It follows that $Z \stackrel{\text { def }}{=} A \cap Y^{1} \subseteq \bigoplus_{i \in I} B_{i}^{1}$ is $\Sigma$-cyclic. In addition, there is an embedding $A / Z \rightarrow Y / Y^{1} \cong \bigoplus_{i \in I} B_{i} / B_{i}^{1}$, so that $A / Z$ is $\Sigma_{f}$-cyclic.

Obviously $\left(\mathrm{b}_{f}\right)$ implies (b), so assume (b) holds and we verify (c). Let $J$ and $K$ be disjoint sets such there are decompositions $Z=\bigoplus_{j \in J}\left\langle x_{j}\right\rangle$ and $A / Z=\bigoplus_{k \in K}\left\langle x_{k}\right\rangle$; let $I=J \cup K$. If $i \in I$, construct a group $B_{i}$ such that $B_{i}^{1}=\left\langle x_{i}\right\rangle$ and $B_{i} / B_{i}^{1}$ is $\Sigma_{f}$-cyclic (such a construction is clearly possible). Since $A / Z$ is $\Sigma_{f}$-cyclic, the isomorphism $Z \rightarrow \bigoplus_{j \in J}\left\langle x_{j}\right\rangle$ extends to a homomorphism $f: A \rightarrow \bigoplus_{j \in J} B_{j} \subseteq \bigoplus_{i \in I} B_{i}$. If $g$ is the composite $A \rightarrow A / Z \subseteq \bigoplus_{k \in K} B_{k} \subseteq \bigoplus_{i \in I} B_{i}$, then it is also easy to verify that $f+g$ : $A \rightarrow Y=\bigoplus_{i \in I} B_{i}$ is the required injection.

We now suppose that (b), $\left(\mathrm{b}_{f}\right)$ and (c) holds and we prove (a). Suppose $0 \rightarrow G \rightarrow X \rightarrow A \rightarrow 0$ is any $L^{2}$-pure short exact sequence. By Corollary 2.9 there is a pull-back diagram

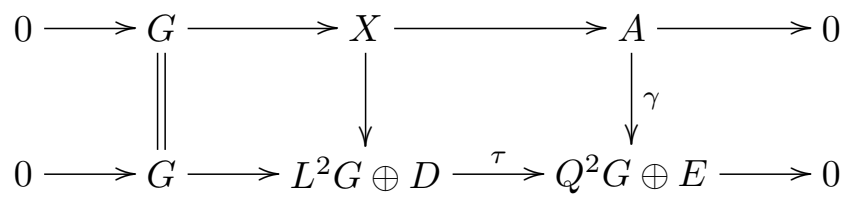

Let $Y=\bigoplus_{i \in I} B_{i} \supseteq A$ be as in (c). Since $Q^{2} G \oplus E$ is divisible, $\gamma$ extends to a homomorphism $g: Y \rightarrow Q^{2} G \oplus E$. Let $g^{1}$ be $g$ restricted to $Y^{1}$. Note that $Y^{1} \cong \bigoplus_{i \in I} B_{i}^{1}$ is $\Sigma$-cyclic. By Corollary 2.7, $0 \rightarrow G^{1} \rightarrow\left(L^{2} G\right)^{1} \oplus D \rightarrow$ $Q^{2} G \oplus E \rightarrow 0$ is pure-exact, and it follow that $g^{1}$ factors as $\tau \circ k^{1}$ for some homomorphism $k^{1}: Y^{1} \rightarrow\left(L^{2} G\right)^{1} \oplus D$.

Now, since $Y / Y^{1} \cong \bigoplus_{i \in I} B_{i} / B_{i}^{1}$ is $\Sigma$-cyclic, it follows that $k^{1}$ extends to a homomorphism $k: Y \rightarrow L^{2} G \oplus D$. 
Consider $g-\tau \circ k: Y \rightarrow Q^{2} G \oplus E$. For every $x \in Y^{1},(g-\tau \circ k)(x)$ $=g^{1}(x)-\tau\left(k^{1}(x)\right)=0$. Therefore, $g-\tau \circ k$ induces a homomorphism $j: Y / Y^{1} \rightarrow Q^{2} G \oplus E$. Since $0 \rightarrow G \rightarrow L^{2} G \oplus D \rightarrow Q^{2} G \oplus E \rightarrow 0$ is pure and $Y / Y^{1}$ is $\Sigma_{f}$-cyclic, $j=\tau \circ h^{\prime}$ for some $h^{\prime}: Y / Y^{1} \rightarrow L^{2} G \oplus D$. Let $h$ be the composition of $h^{\prime}$ with the canonical homomorphism $Y \rightarrow Y / Y^{1}$.

The preceding paragraph implies that $g-\tau \circ k=\tau \circ h$, so $g=\tau \circ(h+k)$. This further implies that $\gamma=\left.g\right|_{A}$ factors through $\tau$. Hence $0 \rightarrow G \rightarrow X \rightarrow$ $A \rightarrow 0$ must split, so that $A$ is $L^{2}$-pure-projective; so (c) implies (a).

We now verify that there are enough $L^{2}$-pure-projectives. Let $A$ be any abelian group. Using a standard construction (see, for example, [6]), we can find a group $M$ such that $M^{2}=A$. Let $\lambda^{1}: U \rightarrow M^{1} \rightarrow 0$ be a pure-projective resolution of $M^{1}$; so $U$ is $\Sigma$-cyclic. It follows that $U$ can be embedded in a group $Y_{0}$ of the form described in (c) such that $U=Y_{0}^{1}$, and that $\lambda^{1}$ can be extended to a homomorphism $\lambda: Y_{0} \rightarrow M$.

Let $Y_{1}$ be a $\Sigma$-cyclic group such that there is a pure-projective resolution $\mu: Y_{1} \rightarrow M \rightarrow 0$ of $M$. Next, let $Y=Y_{0} \oplus Y_{1}$ and $\sigma: Y \rightarrow M$ be $\sigma\left(\left(y_{0}, y_{1}\right)\right)=\lambda\left(y_{0}\right)+\mu\left(y_{1}\right)$.

Clearly, $\sigma$ is surjective and we let $G$ be its kernel. Since for all $n \in \mathbb{N}$ we have $M[n]=\mu\left(Y_{1}[n]\right) \subseteq \sigma(Y[n])$, it follows that $G$ is pure in $Y$. In particular, $G^{1}=G \cap Y^{1}$, from which it follows that there is a left exact sequence

$$
0 \rightarrow G^{1} \rightarrow Y^{1} \rightarrow M^{1} .
$$

However, since $Y^{1}=Y_{0}^{1}=U$, this is, in fact, our pure-projective resolution of $M^{1}$; so the sequence is also right exact, and even pure-exact. This shows that for all $n \in \mathbb{N}$ there is another short exact sequence

$$
0 \rightarrow n G^{1} \rightarrow n Y^{1} \rightarrow n M^{1} \rightarrow 0 .
$$

What we have just shown is that the $\mathbb{Z}^{2}$-adic topology on $G$ is induced by the $\mathbb{Z}^{2}$-adic topology on $Y$. Let $R=\sigma^{-1}(A)=\sigma^{-1}\left(M^{2}\right) \subseteq Y$; so $R$ is the closure of $G \subseteq Y$ in the $\mathbb{Z}^{2}$-adic topology on $Y$. In addition, by the implication (c) $\Rightarrow$ (a), which we have already established, we can conclude that $R \subseteq Y$ is $L^{2}$-pure-projective.

Denote the inclusion $G \subseteq R$ by $\nu$. Since, in the $\mathbb{Z}^{2}$-topology, $G$ is dense in $R$ and $L^{2} G$ is complete, the inclusion $\phi_{G}: G \subseteq L^{2} G$ extends to a homomorphism $f: R \rightarrow L^{2} G$ with $\phi_{G}=f \circ \nu$; in fact this extension is 
unique if we demand that it be continuous with respect to the topology on $R$ induced by the $\mathbb{Z}^{2}$-topology on $Y$. By Proposition 2.4, we can conclude that $0 \rightarrow G \rightarrow R \rightarrow A \rightarrow 0$ is $L^{2}$-pure; and in fact, it is an $L^{2}$-pure-projective resolution of $A$. Therefore, there are enough $L^{2}$-projectives.

Finally, we use the last construction to show that (a) implies (c). If $A$ is $L^{2}$-pure-projective, it follows that $0 \rightarrow G \rightarrow R \rightarrow A \rightarrow 0$ splits, so that $A \subseteq A \oplus G \cong R \subseteq Y$. This completes the proof.

The following two statements are elementary consequences of Theorem 2.10.

Corollary 2.11 If $A$ is $L^{2}$-pure-projective, then any subgroup of $A$ is also $L^{2}$-pure-projective.

Corollary 2.12 If $A$ is $L^{2}$-pure-projective, then its Ulm subgroup $A^{1}$ is S-cyclic.

The next result, which complements Proposition 2.5, is a general categorical consequence of the fact that $L^{2}$-purity has enough injectives and projectives:

Proposition 2.13 Suppose $A$ is a subgroup of $B$ and $B$ is a subgroup of C.

(a) If $A$ is $L^{2}$-pure in $B$ and $B$ is $L^{2}$-pure in $C$, then $A$ is $L^{2}$-pure in $C$.

(b) If $A$ is $L^{2}$-pure in $C$ and $B / A$ is $L^{2}$-pure in $C / A$, then $B$ is $L^{2}$-pure in $C$.

\section{Torsion-Free Groups and Localizations}

If $A$ is any group and $p$ is a prime, let $A_{p}=A \otimes \mathbb{Z}_{p}$ be the localization of $A$ at $p$ (so $\mathbb{Z}_{p}$ consists of the rational numbers with denominators relatively prime to $p$ ). It is readily verified that if $G$ is a $\mathbb{Z}_{p}$-module and we use $\left\{p^{\omega+n} G\right\}_{n<\omega}$ as a neighborhood base of 0 , then we can define the concept of $L_{p}^{2}$-purity, and the obvious translations of the results of the last section continue to hold.

Proposition 3.1 If $A$ is $L^{2}$-pure-projective, then for every prime $p$, the localization $A_{p}$ is $L_{p}^{2}$-projective in the category of $\mathbb{Z}_{p}$-modules.

Proof. Suppose $Z \subseteq A$ is as in Theorem 2.10(b). Then, since $\mathbb{Z}_{p}$ is a flat $\mathbb{Z}$-module, we have a short exact sequence 


$$
0 \rightarrow Z_{p} \rightarrow A_{p} \rightarrow(A / Z)_{p} \rightarrow 0
$$

Since, as $\mathbb{Z}_{p}$-modules, $Z_{p}$ and $(A / Z)_{p}$ are $\Sigma$-cyclic, we can conclude that $A_{p}$ is $L_{p}^{2}$-projective, as required.

Proposition 3.2 A torsion-free $\mathbb{Z}_{p}$-module $A_{p}$ is $L_{p}^{2}$-projective iff it is free.

Proof. Certainly, if $A_{p}$ is free, then it is $L_{p}^{2}$-projective. On the other hand, suppose $A_{p}$ is $L_{p}^{2}$-projective. We can therefore embed $A_{p}$ in a module $Y_{p}=$ $\bigoplus_{i \in I} B_{i}$ as in Theorem 2.10(c). Fix an $i \in I$; since $B_{i}$ is reduced and has torsion-free rank at most 1 , every torsion-free $\mathbb{Z}_{p}$-submodule of $B_{i}$ is free (in fact, cyclic). It follows from a result of Griffith (see [2, Lemma 101.4]) that every torsion-free $\mathbb{Z}_{p}$-submodule of $Y$, such as $A_{p}$, is also free, completing the proof.

Suppose $A$ is a torsion-free group. We say $A$ is locally cyclic if for every prime $p$, the localization $A_{p}$ is a cyclic $\mathbb{Z}_{p}$-module. This is equivalent to requiring that $A$ has rank one and that the type of $A$ is finite at all primes (i.e., $A$ is not $p$-divisible for any prime $p$ ). Similarly, we will say $A$ is locally free if for every prime $p$, the localization $A_{p}$ is a free $\mathbb{Z}_{p}$-module. (This terminology is natural, but for some authors, to say $A$ is locally free means that every finite rank subgroup of $A$ is free, which is a significantly different concept). Recall that a subgroup $Z$ of a torsion-free group $A$ is said to be full-rank if $A / Z$ is torsion.

Theorem 3.3 If A is a torsion-free group, then in the following (a) implies (b) implies (c) implies (d).

(a) A has a full-rank free subgroup $Z$ such that for every prime $p$, the $p$ torsion component of $A / Z$ is finite;

(b) there is a locally cyclic group $\mathbb{P} \subseteq \mathbb{Q}$, and an embedding of $A$ into a direct sum of copies of $\mathbb{P}$.

(c) $A$ is $L^{2}$-pure-projective;

(d) $A$ is locally free.

If, in addition, A has countable rank, then (d) implies (a), so that all of these conditions are equivalent.

Proof. Suppose (a) holds, and $Z \subseteq A$ is as stated. We may assume $A \subseteq$ 
$\bigoplus_{i \in I} \mathbb{Q} e^{i}$, where $Z=\bigoplus_{i \in I} \mathbb{Z} e^{i}$. For each prime $p$, suppose the $p$-torsion of

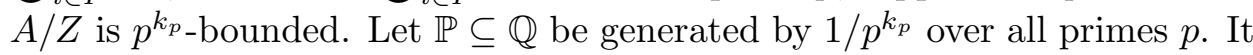
easily follows, then, that $A \subseteq \bigoplus_{i \in I} \mathbb{P} e^{i}$, as stated.

Suppose now that (b) holds. Since the collection of $L^{2}$-pure-projectives is closed under direct sums and subgroups, it suffices to show that $\mathbb{P}$ is $L^{2}$ pure-projective. However, if $Z$ is any non-zero cyclic subgroup of $\mathbb{P}$, then for all primes $p$, the $p$ component of $\mathbb{P} / Z$ will be cyclic, so that $\mathbb{P} / Z$ will be $\Sigma_{f}$-cyclic. Therefore, $\mathbb{P}$ is $L^{2}$-pure-projective, and (c) follows.

Suppose now that (c) holds. If $p$ is an arbitrary prime, then by Proposition $3.1, A_{p}$ is a torsion-free $L_{p}^{2}$-pure-projective. Therefore, by Proposition 3.2 , (d) must hold.

Finally, suppose $A$ has countable torsion-free rank and (d) holds; i.e., $A_{p}$ is a free $\mathbb{Z}_{p}$-module for every prime $p$. We need to verify (a).

Again, let $\left\{e^{k}: k<\omega\right\} \subseteq A$ be chosen so that they are a basis for the rational vector space $\mathbb{Q} \otimes A=\mathbb{Q} A$. Next, let $M^{0}=\{0\}$, and for every $n \in \mathbb{N}$, let $M^{n}$ be the pure hull of $\left\langle e^{k}: k<n\right\rangle$ in $A$; in other words, $M^{n}$ is the intersection of $A$ with the span of $\left\{e^{k}: k<n\right\}$ in $\mathbb{Q} A$. In addition, let $\left\{p_{k}: k<\omega\right\}$ be an enumeration of all the prime numbers.

We will find a second $\mathbb{Q}$-basis $\left\{b^{k}: k<\omega\right\} \subseteq A$ of $\mathbb{Q} A$ such that for every $n \in \mathbb{N}$ we have

(1) $\mathbb{Q}\left\langle b^{k}: k<n\right\rangle=\mathbb{Q}\left\langle e^{k}: k<n\right\rangle$;

(2) For every $k \leq n$ there is a splitting of $\mathbb{Z}_{p_{k}}$-modules $M_{p_{k}}^{n+1}=M_{p_{k}}^{n} \oplus \mathbb{Z}_{p_{k}} b^{n}$.

Proceeding inductively, suppose we have constructed $b^{0}, \ldots, b^{n-1}$. For each $k \leq n$, the $p_{k}$-localization $M_{p_{k}}^{n+1}$ is a finitely generated free $\mathbb{Z}_{p_{k}}$-module. Therefore, $M_{p_{k}}^{n+1} / M_{p_{k}}^{n}$ is isomorphic to a finitely generated $\mathbb{Z}_{p_{k}}$-submodule of $\mathbb{Q}$, so that it is a cyclic $\mathbb{Z}_{p_{k}}$-module. Therefore, $M_{p_{k}}^{n}$ is a summand of $M_{p_{k}}^{n+1}$; so there is an element $a^{k} \in M^{n+1}$ such that $M_{p_{k}}^{n+1}=M_{p_{k}}^{n} \oplus \mathbb{Z}_{p_{k}} a^{k}$.

Find integers $x_{0}, \ldots, x_{n}$ such that $x_{0}+\cdots+x_{n}=1$ and for each $i, j \leq n$, $p_{i} \mid x_{j}$ iff $i \neq j$. We set $b^{n}=x_{0} a^{0}+\cdots+x_{n} a^{n}$. For each $k \leq n$, in the composite homomorphism $\pi: M_{p^{k}}^{n+1} \rightarrow M_{p_{k}}^{n+1} / M_{p_{k}}^{n} \cong \mathbb{Z}_{p_{k}}, \pi\left(a^{k}\right)$ is a unit in $\mathbb{Z}_{p_{k}}$. Since $p_{k} \nmid x_{k}$, it follows that $\pi\left(x_{k} a^{k}\right)$ is also a unit in $\mathbb{Z}_{p_{k}}$. Now, since $b^{n}-x_{k} a^{k} \in p_{k} M_{p_{k}}^{n+1}$, we can conclude that $\pi\left(b^{n}\right) \equiv \pi\left(x_{k} a^{k}\right)$ $(\bmod p)$, so that $\pi\left(b^{n}\right)$ is also a unit in $\mathbb{Z}_{p_{k}}$. This clearly implies $(2)$. Since $\left\langle b^{0}, \ldots, b^{n}\right\rangle \subseteq \mathbb{Q}\left\langle e^{k}: k<n+1\right\rangle$ has rank $n+1$, (1) must hold as well.

Let $Z=\left\langle b^{k}: k<\omega\right\rangle$, which is clearly $\Sigma$-cyclic. Since $A / Z$ and $(A / Z)_{p_{k}}$ have the same $p_{k}$-torsion, the next statement completes the proof of our 
implication.

Claim A: For every prime $p_{k},(A / Z)_{p_{k}}$ is finite.

In fact, by condition (2) of our construction we have

$$
A_{p_{k}} \cong M_{p_{k}}^{k} \oplus\left(\bigoplus_{k \leq n} \mathbb{Z}_{p_{k}} b^{n}\right)
$$

So we can conclude that

$$
(A / Z)_{p_{k}} \cong A_{p_{k}} / Z_{p_{k}} \cong M_{p_{k}}^{k} / \bigoplus_{n<k} \mathbb{Z}_{p_{k}} b^{n} .
$$

Since $M_{p_{k}}^{k}$ is a finitely generated free $\mathbb{Z}_{p_{k}}$-module with $\bigoplus_{n<k} \mathbb{Z}_{p_{k}} b^{n}$ as a free full-rank $\mathbb{Z}_{p_{k}}$-submodule, the claim follows, and hence the proof is complete.

We now verify that the implications in Theorem 3.3 cannot be reversed for torsion-free groups of uncountable rank.

Example 3.4 There is a torsion-free group of uncountable rank that satisfies Theorem 3.3 (b), but not (a).

Proof. Let $\mathbb{P}$ be any torsion-free group that is locally cyclic, but not cyclic, and let $I$ be any uncountable index set. It follows that $A=\bigoplus_{i \in I} \mathbb{P} e^{i}$ trivially satisfies (b). If $Z \subseteq A$ were as in (a), then since $A / Z$ is countable, by a standard "back-and-forth" argument we could find a countable subgroup $X \subseteq A$ such that $Z+X=A, X=\bigoplus_{i \in C} \mathbb{P} e^{i}$ where $C \subseteq I$ is countable, and $X \cap Z$ is also summand of $Z$. It would then follow that

$$
\bigoplus_{i \in I-C} \mathbb{P} e^{i} \cong A / X=(X+Z) / X \cong Z /(X \cap Z) .
$$

But since the right group is free and the left is not, we can conclude that (a) does not hold.

Example 3.5 There is a torsion-free group of uncountable rank that satisfies Theorem 3.3 (c), but not (b).

Proof. Let $\left\{\mathbb{P}_{j}: j \in J\right\}$ be a set of torsion-free locally cyclic groups such 
that every such locally cyclic group is isomorphic to something on the list. Next, let $A=\bigoplus_{j \in J} \mathbb{P}_{j} b^{j}$. Since each $\mathbb{P}_{j}$ is $L^{2}$-pure-projective, so is $A$. Suppose $A$ also satisfies (b) and that $A \subseteq \bigoplus_{i \in I} \mathbb{P} e^{i}$. If $j \in J$ is chosen so that the type of $\mathbb{P}_{j}$ is strictly greater than the type of $\mathbb{P}$, then it follows that $\mathbb{P}_{j} b^{j} \subseteq A$ cannot, in fact, be embedded in $\bigoplus_{i \in I} \mathbb{P} e^{i}$. This contradiction shows that (b) cannot hold.

Finally, to verify that (d) does not necessarily imply (c), we actually employ a bit of set theory. It is plausible that this can be avoided, but even so, the example certainly shows that it is pointless to attempt to prove that (d) implies (c) for groups of uncountable rank.

Theorem $3.6(V=L) \quad$ Assuming the axiom of constructibility, there is a torsion-free group that is locally free, but not $L^{2}$-pure-projective.

Proof. Let $A$ be any set of cardinality $\aleph_{1}$ and $\left\{M^{i}\right\}_{i<\omega_{1}}$ be a filtration of $A$ such that for all $i, M^{i+1}-M^{i}$ is a countably infinite set. We will construct the group $A$ by imposing on each $M^{i}$ a group structure. In fact, if $\left\{e^{\ell}\right\}_{\ell<\omega_{1}}$ is the standard basis for $V=\bigoplus_{\ell<\omega_{1}} \mathbb{Q} e^{\ell}$, then in our construction we will identify $A$ with a subgroup of $V$ so that for all $i<\omega_{1}$ we have

$$
M^{i}=A \cap\left(\bigoplus_{\ell<i} \mathbb{Q} e^{\ell}\right) \stackrel{\text { def }}{=} A \cap V^{i} .
$$

This will follow if in our construction we make sure that for every $i \leq j<\omega_{1}$ we have

$$
M^{i}=M^{j} \cap V^{i}
$$

To begin, let $\mathbb{P}$ be the subgroup of $\mathbb{Q}$ generated by $1 / p$ for all primes $p$. We are going to start with $M^{0}=\{0\}$ and $M^{1}=\mathbb{P} e^{0}$.

So suppose we have constructed $M^{i}$ for all $i<j<\omega_{1}$ and we wish to construct $M^{j}$. Certainly, if $j$ is a limit, then the smoothness of filtrations determines our group structure on $M^{j}$. And since the equations $(i, k)$ hold whenever $i \leq k<j$, it easily follows that each equation $(i, j)$ also holds for $i \leq j$.

It remains to define our operation on $M^{j} \subseteq V^{j}$ when $j=k+1$ is isolated. We will do so in such a way that if we restrict the projection $V^{j} \rightarrow \mathbb{Q} e^{k}$ to $M_{j}$, the result is a short exact sequence 


$$
0 \rightarrow M^{k} \rightarrow M^{k+1} \rightarrow \mathbb{P} e^{k} \rightarrow 0
$$

Before continuing, we note that whenever $i \leq j$, equation $(i, j)$ will continue to hold: Clearly if $i=j$, then equation $(i, j)$ trivially follows, and if $i<j$, then $i \leq k$. So, since we are assuming $(i, k)$ holds and sequence $(k)$ is exact, we can conclude that

$$
M^{i}=M^{k} \cap V^{i}=M^{j} \cap V^{k} \cap V^{i}=M^{j} \cap V^{i} .
$$

We now show how to define $M^{j}=M^{k+1}$ by splitting the argument into two cases. This is where we will employ $\mathrm{V}=\mathrm{L}$. By the diamond principle $(\diamond)$, which holds in the constructible universe, there is a collection of subsets $L^{i} \subseteq M^{i}$ for $i<\omega_{1}$, such that if $Z \subseteq A$, then there is a stationary set of ordinals $i$ such that $Z \cap M^{i}=L^{i}$.

CASE 1: $L^{k}$ is a free, full-rank subgroup of $M^{k}$ such that $M^{k} / L^{k}$ is $\Sigma_{f^{-}}$ cyclic.

CAse 2: Case 1 does not hold.

For simplicity we start with Case 2: Here we just let $M^{j}=M^{k} \oplus \mathbb{P} e^{k} \subseteq$ $V^{j}$. This clearly means that sequence $(k)$ is (splitting) exact.

So, suppose we are in Case 1. Observe first that $M^{1}=\mathbb{P} e^{0} \cong \mathbb{P}$ is contained in $M^{k}$. Since $M^{1} \cap L^{k} \subseteq L^{k}$ will be cyclic and $M^{1} /\left(M^{1} \cap L^{k}\right)$ embeds in $M^{k} / L^{k}$, it follows that for an infinite number of primes $p$, the $p$-torsion component of $M^{k} / L^{k}$ is non-zero (and $\Sigma_{f}$-cyclic); let $\mathcal{D}^{k}$ be the collection of primes where this holds.

For each prime $p$ define an element $g_{p} \in V^{j}$ as follows: If $p \in \mathcal{D}^{k}$, let $w_{p} \in M^{k}$ be chosen so that $w_{p}+L^{k}$ is an element of $M^{k} / L^{k}$ whose $p$-height is 0 (i.e., it is not divisible by $p$ in this quotient group); and if $p \notin \mathcal{D}_{k}$, let $w_{p}=0$. We now let

$$
g_{p}=(1 / p)\left(w_{p}+e^{k}\right) \in V^{k} \oplus \mathbb{Q} e^{k}=V^{j}
$$

We now identify $M^{j}$ with

$$
M^{k}+\left\langle g_{p}: p \text { is a prime }\right\rangle
$$

so $e^{k}=p g_{p}-w_{p} \in M^{j}$. Considering the sequence $(k)$, under the projection $V^{j} \rightarrow \mathbb{Q} e^{k}$ we have $g_{p} \mapsto(1 / p) e^{k}$, so that the image of this map restricted 
to $M^{j}$ is clearly $\mathbb{P} e^{k}$. So we need to show that $M^{k}=M^{j} \cap V^{k}$. Clearly $M^{k} \subseteq M^{j} \cap V^{k}$. Conversely, let $u \in M^{j} \cap V^{k}$. Since $u \in M^{j}$, there is a $v \in M^{k}$, distinct primes $p_{1}, \ldots, p_{n}$ and integers $x_{1}, \ldots, x_{n}$ such that

$$
\begin{aligned}
u & =v+x_{1} g_{p_{1}}+\cdots+x_{n} g_{p_{n}} \\
& =v+\left(x_{1} / p_{1}\right) w_{p_{1}}+\cdots+\left(x_{n} / p_{n}\right) w_{p_{n}}+\left(x_{1} / p_{1}+\cdots+x_{n} / p_{n}\right) e^{k}
\end{aligned}
$$

Since $u \in V^{k}$, we can conclude that

$$
x_{1} / p_{1}+\cdots+x_{n} / p_{n}=0,
$$

but this readily implies that $x_{1} / p_{1} \in \mathbb{Z}, \ldots, x_{n} / p_{n} \in \mathbb{Z}$. Therefore,

$$
u=v+\left(x_{1} / p_{1}\right) w_{p_{1}}+\cdots+\left(x_{n} / p_{n}\right) w_{p_{n}} \in M^{k},
$$

as required.

We have $A=\cup_{j<\omega_{1}} M^{j}$. We next observe that the exact sequences $(k)$ guarantee that $A$ is locally free: If $p$ is a prime, then for all $k<\omega_{1}$ we will have a short exact sequence of localizations

$$
0 \rightarrow M_{p}^{k} \rightarrow M_{p}^{k+1} \rightarrow \mathbb{P} e^{k} \otimes \mathbb{Z}_{p} \rightarrow 0
$$

But since $\mathbb{P} e^{k} \otimes \mathbb{Z}_{p} \cong \mathbb{Z}_{p}$ is a cyclic $\mathbb{Z}_{p}$-module, all of these sequences split, showing that $A_{p} \cong \bigoplus_{k<\omega_{1}} \mathbb{Z}_{p}$ is free.

To complete the proof, we need to show that $A$ fails to be $L^{2}$-pureprojective. Assuming otherwise, by Theorem $2.10\left(\mathrm{~b}_{f}\right) A$ has a $\Sigma$-cyclic subgroup $Z$ such that $A / Z$ is $\Sigma_{f}$-cyclic; so $Z$ is a free, full-rank subgroup of $A$. Since $\left\{M^{i}\right\}_{i<\omega_{1}}$ is a filtration of $A$, there is a closed and unbounded subset $\mathcal{C} \subseteq \omega_{1}$, such that for every $k \in \mathcal{C},\left(M^{k}+Z\right) / Z$ is a summand of $A / Z$.

By the diamond principle $(\diamond)$, there is a $k \in \mathcal{C}$ such that $Z \cap M^{k}=L^{k}$. Since

$$
M^{k} / L^{k}=M^{k} /\left(M^{k} \cap Z\right) \cong\left(M^{k}+Z\right) / Z \subseteq A / Z,
$$

$L^{k}$ is a free full-rank subgroup of $M^{k}$ and $M^{k} / L^{k}$ is $\Sigma_{f}$-cyclic.

So $j=k+1$ must be in the above Case 1 of the construction of $A$. We will obtain a contradiction that proves $A$ is not $L^{2}$-pure-projective by 
showing that our construction in that Case ensures that $\left(M^{k}+Z\right) / Z$ cannot, in fact, be a summand of $A / Z$.

Since $A / Z$ is torsion, $m e^{k} \in Z$ for some non-zero integer $m$. Looking back at the notation of Case 1 , since the set of primes $\mathcal{D}^{k}$ is infinite and $m$ has at most a finite number of prime divisors, there is a $p \in \mathcal{D}^{k}$ relatively prime to $m$. Consider the element $w_{p}+L^{k}$ in that argument, which was assumed to be an element of $M^{k} / L^{k}$ of $p$-height 0 . Since $m$ is relatively prime to $p, m w_{p}+L^{k} \in M^{k} / L^{k} \cong\left(M^{k}+Z\right) / Z$ will also have $p$-height 0 . However, in $A / Z$ we have

$$
p m g_{p}+Z=p m(1 / p)\left(w_{p}+e^{k}\right)+Z=m w_{p}+m e^{k}+Z=m w_{p}+Z .
$$

This means that, in fact, $m w_{p}+Z$ has a greater $p$-height in $A / Z$ than in $\left(M^{k}+Z\right) / Z$. So $\left(M^{k}+Z\right) / Z$ cannot actually be a summand of $A / Z$, which gives our contradiction and completes the proof.

\section{Countable Groups of Torsion-Free Rank One}

If $p$ is a prime, $A$ is a group and $x \in A$, we will use the notation $|x|_{p}$ for the $p$-height of $x$ in $A$.

Theorem 4.1 Suppose $A$ is a countable group of torsion-free rank one. Then $A$ is $L^{2}$-pure-projective iff (a) $A^{2}=\{0\}$, (b) there is a (non-torsion) element $x \in A$ such that $\left|p^{k} x\right|_{p} \leq \omega+k$ for all primes $p$ and all $k<\omega$.

Proof. Suppose first that $A^{2}=\{0\}$ and we can find such an element $x$; let $X=\langle x\rangle$. Observe that $A / X$ is torsion, and we begin by showing that it suffices to assume that $A / X$ is a $p$-group: If $T_{p} / X$ is the $p$-torsion component of $A / X$, then there is a decomposition $A / X=\bigoplus_{p}\left(T_{p} / X\right)$. Since all heights computed in $T_{p}$ are no larger than when computed in $A$, (a) and (b) also holds in $T_{p}$. Suppose for each prime $p$ we can find a $\Sigma_{f}$-cyclic subgroup $Y_{p} \subseteq T_{p}$ such that $X \cap Y_{p}=\{0\}$ and $T_{p} /\left(X \oplus Y_{p}\right)$ is $\Sigma_{f}$-cyclic. It easily follows that there is an (internal) direct sum $Z \stackrel{\text { def }}{=} X \oplus\left(\bigoplus_{p} Y_{p}\right) \subseteq A$, so that $Z$ is $\Sigma$-cyclic, and that

$$
A / Z \cong \bigoplus_{p}\left\{T_{p} /\left(X \oplus Y_{p}\right)\right\}
$$

is $\Sigma_{f}$-cyclic. So there is no loss of generality in assuming that $A / X$ is, in 
fact, a $p$-group for some prime $p$. This clearly also means that $A$ can have no $q$-torsion for any prime $q \neq p$.

Next, we verify that $p^{\omega 2} A=\{0\}$ : Suppose $a \in p^{\omega 2} A$. If $a$ has infinite order, then $m a=n p^{k} x$ for some $m, n, k$ with $(n, p)=1$. This gives the following contradiction:

$$
\omega 2 \leq|a|_{p} \leq|m a|_{p}=\left|n p^{k} x\right|_{p}=\left|p^{k} x\right|_{p} \leq \omega+k
$$

Therefore, the order of $a$ must be a power of $p$. But this would then imply that $a \in q^{\omega 2}$ for all primes $q \neq p$; and so $a \in A^{2}=\{0\}$. Hence $p^{\omega 2} A=\{0\}$.

We now claim that $X$ is a summand of $Z \stackrel{\text { def }}{=} X+p^{\omega} A$. First, observe that $X \cap p^{\omega} A$ is either $\{0\}$ or an infinite cyclic subgroup of $p^{\omega} A$; in particular, $X \cap p^{\omega} A$ is nice in $p^{\omega} A$ (see, for example, [2, Lemma 104.1]). Because $p^{\omega}\left(p^{\omega} A\right)=p^{\omega 2} A=\{0\}$, we can conclude that

$$
p^{\omega} A /\left(X \cap p^{\omega} A\right) \cong\left(X+p^{\omega} A\right) / X=Z / X \quad(\subseteq A / X)
$$

is a countable $p$-group with no elements of infinite $p$-height. Therefore, this quotient is $\Sigma_{f}$-cyclic, and in particular, a totally projective $p$-group.

Since we are assuming that $\left|p^{k} x\right|_{p} \leq \omega+k$ for all $k$, we can conclude the identity map on

$$
p^{\omega} A \cap X \quad\left(\subseteq p^{\omega} A\right) \rightarrow p^{\omega} A \cap X \quad(\subseteq X)
$$

does not decrease heights (computed in $p^{\omega} A$ and $X$, respectively). Therefore, (as $p^{\omega} A \cap X$ is nice in $p^{\omega} A$ and $p^{\omega} A /\left(p^{\omega} A \cap X\right)$ is totally projective) this identity map extends to a homomorphism $\phi: p^{\omega} A \rightarrow X$ (see [2, Corollary 81.4]). Since we can extend $\phi$ to a homomorphism $Z=X+p^{\omega} A \rightarrow X$ by letting it be the identity on $X$, we can conclude that $X$ is actually a summand of $Z$. Say $Z=X \oplus Y$; so $Y \cong Z / X$ is $\Sigma_{f}$-cyclic and $Z$ is $\Sigma$-cyclic.

We now want to show that $A / Z$ is also $\Sigma_{f}$-cyclic. Note that it is the homomorphic image of $A / X$, so it is a $p$-group. In addition, $A / p^{\omega} A$ has no elements of infinite $p$-height, and it has $Z / p^{\omega} A=\left(X+p^{\omega} A\right) / p^{\omega} \cong X /(X \cap$ $\left.p^{\omega} A\right)$ as a cyclic, and therefore nice, subgroup. So $A / Z \cong\left(A / p^{\omega} A\right) /\left(Z / p^{\omega} A\right)$ will be a countable $p$-group with no elements of infinite $p$-height, i.e., $A / Z$ is $\Sigma_{f}$-cyclic.

So one direction is complete. We now consider the converse. Note that (a) certainly holds whenever $A$ is any $L^{2}$-pure-projective. Regarding (b), 
suppose $Z$ is a $\Sigma$-cyclic subgroup of $A$ and $A / Z$ is $\Sigma_{f}$-cyclic. Let $\langle u\rangle$ be an infinite cyclic summand of $Z$.

Claim A: The set

$$
\mathcal{P}=\left\{p \text { is a prime such that }\left|p^{k} u\right|_{p}>\omega+k \text { for some } k<\omega\right\}
$$

is finite.

Assume otherwise. For every $p \in \mathcal{P}$, choose $k<\omega$ and $w_{p} \in p^{\omega} A$ such $p^{k+1} w_{p}=p^{k} u$. Observe that $w_{p}+Z$ will be a $p$-torsion element of $A / Z$ (since $\left.p^{k+1}\left(w_{p}+Z\right)=0\right)$. In addition, it has infinite $p$-height (since $w_{p} \in p^{\omega} A$ ). Since $A / Z$ is $\Sigma_{f}$-cyclic, this means that $w_{p}+Z=0$, i.e., $w_{p} \in Z$.

We can conclude that $W=\left\langle u, w_{p}: p \in \mathcal{P}\right\rangle$ is a subgroup of $Z$; so in particular, $W$ is $\Sigma$-cyclic. Therefore, if $S$ is the torsion subgroup of $W$, then $W / S$ is cyclic. However, it is easily seen that $W / S$ is actually isomorphic to the subgroup of $\mathbb{Q}$ generated by $1 / p$ for all $p \in \mathcal{P}$ (using the assignment $\left.w_{p} \mapsto 1 / p\right)$. Since the latter group fails to be cyclic (because $\mathcal{P}$ is infinite), we have our contradiction, and Claim A follows.

Claim B: If $p \in \mathcal{P}$, then the $p$-height sequence of $u$ has only a finite number of gaps.

To see this we first note that the localization $A_{p}$ must be $L_{p}^{2}$-pureprojective. Since $p \in \mathcal{P}$, for some $k<\omega, p^{k} u$ is an element of the Ulm subgroup $A_{p}^{1}$. And since $A_{p}^{1}$ is a $\Sigma$-cyclic $\mathbb{Z}_{p}$-module, the $p$-height sequence of $p^{k} u \in A_{p}^{1}$ can only have finitely many gaps, as stated.

So by Claim A we may write $\mathcal{P}=\left\{p_{1}, \ldots, p_{m}\right\}$. By Claim B, for each $n \leq m$, there is a $j_{n}<\omega$ such that the $p_{n}$-height sequence of $p_{n}^{j_{n}} u$ has no gaps. Let $\left|p_{n}^{j_{n}} u\right|_{p_{n}}=\omega+\ell_{n}\left(\right.$ so $\left.\ell_{n}>j_{n}\right)$ and let

$$
s=p_{1}^{j_{1}} \cdots p_{m}^{j_{m}} \text { and } t=p_{1}^{\ell_{1}} \cdots p_{m}^{\ell_{m}}
$$

Since for each $n \leq m,|s u|_{p_{n}}=\omega+\ell_{n}$, by the Chinese Remainder Theorem we can find an $x \in A$ such that $s u=t x$, where $|x|_{p_{n}}=\omega$ for each $n \leq m$.

It follows that for every $p \in \mathcal{P}$, the $p$-height sequence of $x$ is simply $(\omega, \omega+1, \omega+2, \ldots)$; while for every $p \notin \mathcal{P}$, the $p$-height sequences of $x$ and $u$ agree. In particular, this implies that $\left|p^{k} x\right|_{p} \leq \omega+k$ for all primes $p$ and all $k<\omega$, as required. 
The following example shows that the obvious generalization of Theorem 4.1 to countable groups of higher torsion-free ranks does not, in fact, hold.

Example 4.2 There is a countable group $A$ of torsion-free rank 2 such that (a) $A^{2}=\{0\}$, (b) for all (non-torsion) elements $x \in A$, all primes $p$ and all $k \in \omega$, we have $\left|p^{k} x\right|_{p} \leq \omega+k$, but $A$ is not $L^{2}$-pure-projective.

Proof. If $q$ is some fixed prime and $U=\left\langle u_{1}\right\rangle \oplus\left\langle u_{2}\right\rangle$ is any free, rank 2 subgroup of the $q$-adic integers $J_{q}$, then let $A$ be defined by the condition that $A / U$ is the $q$-torsion subgroup of $J_{q} / U$. It follows that $A$ is $q$-pure in $J_{q}$, so that $A / q A$ embeds in $J_{q} / q J_{q} \cong \mathbb{Z} /(q)$. This implies that $A_{q}$ is not a free $\mathbb{Z}_{q}$-module. So $A$ is not locally free, which means that it is not $L^{2}$-pure-projective.

We claim that $p^{\omega} A=\{0\}$ for all primes $p$. If $p=q$, then this follow since $q^{\omega} J_{q}=\{0\}$. Suppose then that $p \neq q$. If $\mathbb{P}$ is the rank one subgroup of $\mathbb{Q}$ generated by $1 / q^{n}$ for all $n \in \mathbb{N}$, it readily follows that there is an embedding $A \subseteq \mathbb{P} u_{1} \oplus \mathbb{P} u_{2} \stackrel{\text { def }}{=} H$. Since $p^{\omega} H=\{0\}$, it follows that $p^{\omega} A=\{0\}$, as claimed.

In particular, it immediately follows from the last paragraph that conditions (a) and (b) are fulfilled, completing the construction.

By Theorem 3.3, the converse of Proposition 3.1 holds for countable torsion-free groups. On the other hand, the next example shows that it does not hold for countable mixed groups.

Example 4.3 There is a countable group $A$ such that for any prime $p$, the localization $A_{p}$ is $L_{p}^{2}$-projective in the category of local $\mathbb{Z}_{p}$-modules, but $A$ is not $L^{2}$-pure-projective.

Proof. Let $\mathbb{P}$ be the subgroup of the rational numbers generated by $\{1 / p: p$ is a prime $\}$. Construct a group $A$ such that $A^{1}=\mathbb{P}$ and $A / \mathbb{P}$ is $\Sigma_{f}$-cyclic. Since $\mathbb{P}$ is locally cyclic, for any prime $p, \mathbb{P}_{p}$ and $(A / \mathbb{P})_{p}$ are $\Sigma$-cyclic $\mathbb{Z}_{p^{-}}$ modules; so $A_{p}$ is $L_{p}^{2}$-projective. On the other hand, since $A^{1}=\mathbb{P}$ is not $\Sigma$-cyclic, $A$ fails to be $L^{2}$-pure-projective.

We next show that the analogue of Proposition 3.1 for $L^{2}$-pure-injective groups does not hold.

Example 4.4 There is an $L^{2}$-pure-injective group $G$ and a prime $p$ such that the localization $G_{p}$ is not $L_{p}^{2}$-pure-injective. 
Proof. Let $G$ be any group such that $p^{\omega} G \cong \mathbb{Z}$ and $G / p^{\omega} G$ is a direct sum of cyclic $p$-groups. Let $T$ be the $p$-torsion subgroup of $G$. Observe that $G / T$ is isomorphic to the subgroup of $\mathbb{Q}$ generated by $1 / p^{n}$ for all $n \in \mathbb{N}$. Therefore, if $q \neq p$ is another prime, then $q^{\omega}(G / T)=\{0\}$; so $q^{\omega} G$ agrees with the $p$-torsion subgroup of $G$. This implies that $G^{1}=0$; and in particular, $G$ is discrete, and hence complete, in the $\mathbb{Z}^{2}$-adic topology.

On the other hand, $p^{\omega} G_{p} \cong \mathbb{Z}_{p}$. Therefore, $G_{p}$ is not complete in the $\mathbb{Z}_{p}^{2}$-adic topology, so that it is not $L_{p}^{2}$-pure-injective.

We finish by showing that the converse of the last example fails as well.

Example 4.5 There is a group $G$ that is not $L^{2}$-pure-injective, but whose localization $G_{p}$ is $L_{p}^{2}$-pure-injective for every prime $p$.

Proof. For every prime $p$, let $H^{p}$ be any $p$-group such that $p^{\omega} H^{p}$ is cyclic of order $p$. If $G=\bigoplus_{p} H^{p}$, then for every prime $p, G_{p} \cong H^{p}$. Since $p^{\omega} H^{p}$ is clearly complete in the $p$-adic topology, $G_{p}$ will be $L_{p}^{2}$-pure-injective. However, since $G^{1}=\bigoplus_{p} \mathbb{Z} /(p)$ is not complete in the $\mathbb{Z}$-adic topology, $G$ is not $L^{2}$-pure-injective.

\section{References}

[1] Eklof P. and Mekler A., Almost Free Modules, Set Theoretic Methods (Revised Edition), North-Holland, Amsterdam, 2002.

[ 2 ] Fuchs L., Infinite Abelian Groups, Volumes I \& II, Academic Press, New York, 1970 and 1973.

[3] Griggith P., Infinite Abelian Group Theory, The University of Chicago Press, Chicago and London, 1970.

[ 4 ] Keef P., Variations of purity in abelian group theory. J. Pure Appl. Algebra. 144(3) (1999), 255-276.

[ 5 ] Keef P., On subgroups of totally projective primary abelian groups and direct sums of cyclic groups, in Groups and model theory, Contemp. Math., 576, Amer. Math. Soc., Providence, RI (2012), 205-215.

[ 6 ] Richman F. and Walker E., Valuated groups. J. Algebra. 56(1) (1979), 145167.

Department of Mathematics

Whitman College

Walla Walla, WA, 99362, USA

E-mail: keef@whitman.edu 